Rev. Saúde públ., S. Paulo, 19:251-62, 1985.

\title{
MORTALIDADE EM RECIFE: APLICAÇÃO DE UM MODELO DE RISCOS COMPETITIVOS*
}

Neir Antunes Paes**

\begin{abstract}
PAES, N.A. Mortalidade em Recife: aplicação de um modelo de riscos competitivos. Rev Saúde públ., S. Paulo, $19: 251-62,1985$.

RESUMO: E apresentada a metodologia de Chiang para a construção de Tábuas de Vida de Múltiplo Decremento, apliçadas à população residente do município de Recife, em 1979, para se avaliar a magnitude de incidência de alguns grupos de causas de morte, segundo a probabilidade de morte, sobrevivência e esperança de vida. A média de vida encontrada para os homens foi de 55,43 anos e para as mulheres foi de 62,41 anos. A eliminação total dos grupos de causas como fatores de risco de morte, propiciou os seguintes ganhos na esperança de vida, para homens e mulheres, respectivamente: doenças infecciosas e parasitárias (7,9 e 8,1 anos), neoplasmas malígnos (6,0 e 6,6 anos), doenças do apareiho circulatório (10,4 e 10,8 anos), doenças do aparelho respiratório (6,5 e 6,7 anos) e causas externas (7,0 e 5,2 anos). Concluiu-se que o nível de saúde do município de Recife refletiu, em 1979, um quadro social e econômico não homogêneo, com uma alta mortalidade por doenças degenerativas, características de regiöes desenvolvidas e, também, por doenças infecciosas e parasitárias, próprias de regiões pouco desenvolvidas.
\end{abstract}

UNITERMOS: Mortalidade, Recife, PE, Brasil. Tábuas de vida, múltiplo decre. mento. Riscos competitivos. Esperança de vida.

\section{INTRODUÇÃO}

O surgimento da causa ou causas de morte no homem se dá pela sua exposição permanente ao risco de contrair uma ou mais enfermidades. Esta capacidade que têm os riscos de se concretizarem no homem, nos permite conceber uma "competição" entre eles, na qual os indivíduos, por seu grau natural de sensibilidade, interagem com os riscos de morte, adquirindo um ou mais deles.

$O$ interesse do homem pela vida e pela morte levou-o a pesquisar esta relação de causa e efeito. Com isto, surgiu a preocupação de como postergar a vida, ou seja, qual seria o efeito produzido, na vida do homem, pela eliminação parcial ou total de certo risco competitivo.

Assim, a teoria dos riscos competitivos aplica-se com o propósito de inves- tigar dados estatísticos vitais, para situaçôes nas quais os homens estão expostos ao risco de morrer por várias causas de morte.

Um dos recursos formais mais utilizados para investigar esta teoria é conhecido como Tábua de Vida de Múltiplo Decremento. A importância deste instrumento decorre de sua aplicabilidade, que atende não somente a objetivos de saúde, como também à alocação de recursos e avaliação de programas de saúde.

A teoria dos riscos competitivos foi preocupação de vários investigadores e entre eles destacamos o modelo não-paramétrico, para a construção de Tábuas de Vida de Múltiplo Decremento, desenvolvido por Chiang ${ }^{3}$, em 1968. Este autor (Chiang) considera a morte de ca-

\footnotetext{
- Resumo de um segmento da dissertação "Tábuas de vida de múltiplo decremento - o modelo de Chiang", apresentada ao Instituto de Matemática e Estatística da Universidade de São Paulo, em 1982, para a obtenção do título de Mestre.

Bolsista da Coordenação de Aperfeiçoamento de Pessoal de Nível Superior - CAPES.

- Rua Gustavo Pinto, 251, Jardim Säo Paulo - 50.000- Recife, PE - Brasil.
} 
PAES, N.A. Mortalidade em Recife: aplicação de um modelo de riscos competitivos. Rev. Saúde públ., S. Paulo, 19:251-62, 1985.

da indivíduo como consequiência de uma única causa básica e que os vários riscos de morte atuam simultaneamente sobre cada indivíduo, havendo para cada risco uma correspondente força de mortalidade. O modelo supõe, ainda, que a força de mortalidade de uma causa de morte não é afetada pela remoção de uma outra. Quanto a esta remoção, ele admite uma eliminação total do risco de morte.

O modelo de Chiang tem suas limitações, que são reconhecidas pelo próprio autor ${ }^{*}$. Uma das críticas formuladas diz respeito ao suposto de que à morte seria atribuída uma única causa básica, e esta suposição é considerada insatisfatória por alguns autores ${ }^{9,13}$, pois a morte de um indivíduo pode ser devida à interação de vários riscos. Para outros estudiosos ${ }^{11,19}$, podemos estar muito mais interessados em conhecer o resultado da eliminação parcial de uma certa causa específica de morte, por estar mais próximo das possibilidades médicas atuais, do que a eliminação total da causa.

Não obstante as críticas formuladas, a importância do modelo de Chiang é destacada por vários autores ${ }^{10,18}$, por sua praticidade, operacionalidade e validade dos seus supostos.

Neste estudo, o problema consiste em medir o efeito causado pela eliminação total de um ou mais riscos de morte sobre a estrutura de mortalidade, por idade e sexo, de uma determinada população, supondo que cada indivíduo desta população está sujeito a $\mathrm{K}(\mathrm{K} \geqslant 2)$ riscos de óbitos competindo pela sua vida. Este efeito, causado pela eliminação total dos riscos de morte, é avaliado pela sua atuação nas probabilidades de morte, de sobrevivência e nas esperanças de vida.

O objetivo deste estudo é construir Tábuas de Vida de Múltiplo Decremento, segundo o modelo de Chiang, para a população residente no município do Recife, em 1979, para se verificar o efeito que se produzirá na mortalidade, de- pois da eliminação total dos seguintes grupos de causas de morte: doenças infecciosas e parasitárias, neoplasmas malígnos, doenças do aparelho circulatório, doenças do aparelho respiratório e causas externas.

Este trabalho justifica-se pela preocupação e pelo interesse em ampliar o conhecimento sobre a duração de vida dos indivíduos, no caso específico, da população residente no município de Recife, trazendo, assim, novos elementos, seja como contribuição ao conhecimento da mortalidade da população brasileira, an tomar Recife como parte dessa totalidade, seja ao explorar mais o saber que se tem da duração e da qualidade de vida da população recifense.

Embora outros trabalhos já tenham sido desenvolvidos no Brasil, no âmbito das mesmas preocupações, este se situa na investigação de mais uma realidade particular, que é a do Recife. No Brasil, o primeiro trabalho que fez uso da metodologia de Chiang, foi o de Gotlieb ${ }^{8}$ (1981). A autora aplicou o modelo para a população residente no município de São Paulo em 1970, com o propósito de avaliar a magnitude de atuação dos grandes grupos de causas de morte. Seu trabalho guarda com o nosso, portanto, a mesma fundamentação teórica e identidade metodológica.

Os resultados e as conclusões a que se chegou neste trabalho, para o município do Recife, são muito semelhantes aos encontrados por Gotlieb para o município de São Paulo. Apesar destes dois centros do país possuírem características sociais e econômicas distintas, a ordem de importância dos grupos de causas e seus diferenciais por setores da população não apresentaram diferenças significativas.

\section{MATERIAL E METODOS}

\section{Dados utilizados}

A população residente no município de Recife, em $1 .^{\circ}$ de julho de 1979 , foi 
PAES, N.A. Mortalidade em Recife: aplicação de um modelo de riscos competitivos. Rev. Saúde públ., S. Paulo, 19:251-62, 1985.

estimada através do método de interpolação geométrico das populações totais dos recenseamentos de $1970^{\circ}$ e $1980^{7}$. Supusemos que a população cresceu em ritmo geométrico, registrando um crescimento mensal de $\mathrm{r}=0,106 \%$ de onde foi estimado o total de 1.186 .973 habitantes para 1979.

Os dados de óbitos referem-se ao ano de 1979*. Os registros de óbitos, em fita magnética, tiveram seu início de 1977 , o que justifica a não utilização, na prática, de dados pré-censitário, censitário e pós-censitário. Até 1978, esses óbitos foram organizados e codificados pela Fundação de Saúde Amaury de Medeiros (FUSAM) - Pernambuco, segundo a 8. ${ }^{a}$ Revisão da Classificação Internacional de Doenças (1960) e os óbitos para 0 ano de 1979 foram tratados pela $9 .^{\text {a }}$ Revisão, de modo que não houve um critério único de classificação no triênio. Tomamos, então, a decisão de trabalhar apenas com o ano de 1979, agrupando as causas segundo a Lista Brasileira para Mortalidade - CID-BR (1980) ${ }^{12}$.

\section{Metodologia}

A metodologia da Tábua de Vida de Múltiplo Decremento requer o conhecimento de diferentes tipos de probabilidades associadas ao intervalo de idade $\left(x_{1}, x_{i}+{ }_{1}\right)$ e que são definidas a seguir:

$\mathrm{p}_{\mathrm{i}}=$ probabilidade de um individuo vivo na idade $x_{1}$ sobreviver ao intervalo $\left(x_{1}, x_{1}+1\right)$.

$q_{i}=1-p_{i}=$ probabilidade de um indivíduo vivo na idade $x_{1}$ morrer no intervalo $\left(x_{1}, x_{1}+{ }_{1}\right)$.

$\mathrm{Q}_{\mathrm{ij}}=$ probabilidade de um indivíduo vivo na idade $x_{1}$ morrer no intervalo $\left(\mathrm{x}_{1}, \mathrm{x}_{1}+1\right)$ devido à causa (ou grupo de causas) $R_{\mathfrak{i}}$ ńa pre- sença de todos os outros riscos de morte atuando na população; chamada de PROBABILIDADE. BRUTA.

$\mathrm{q}_{\mathrm{i} j}=$ probabilidade de um indivíduo vivo na idade $x_{1}$ vir a morrer no intervalo $\left(x_{1}, x_{1}+{ }_{1}\right)$ se $R_{1}$ é eliminado como um risco de morte; chamada de PROBABILIDADE LIQUIDA.

Chiang, para estimar estes parâmetros, admitiu alguns supostos básicos como a identidade das forças de mortalidade $\mu_{1}(t)$, sendo a soma destas forças igual à força total $\mu(\mathrm{t})$ e que o quociente $\left(\mu_{1}(\mathrm{t}) / \mu(\mathrm{t})\right)=\mathrm{k}_{\mathrm{i}}$ seja uma constante em cada intervalo de idade.

Como resultado dos supostos básicos ele pode estimar, através de uma série de relações matemáticas, as probabilidades bruta e líquida de morte.

O parâmetro de interesse, no contexto do nosso trabalho, consiste em estimar a probabilidade líquida de morte, que não é observável para uma população humana, sendo obtida, somente, mediante sua relação com a probabilidade bruta.

Esta relação, Chiang expressou como' $\mathrm{q}_{\mathrm{i} j}=1-\mathrm{p}_{\mathrm{i}}^{\left[\left(\mathrm{q}_{\mathrm{i}}-\mathrm{Q}_{\mathrm{j}}\right) / \mathrm{q}_{\mathrm{i}}\right]}$

Mediante a teoria de estimação de parâmetros, Chiang encontrou que o estimador que $q_{i j}$ é dado por:

$$
\begin{aligned}
\hat{q}_{1 j}=1-p_{1}\left[\left(D_{1}-D_{i j}\right) / D_{i}\right] & \\
i & =0,1, \ldots, \text { anos } \\
j & =1,2, \ldots, r, \text { causas }
\end{aligned}
$$

onde

$$
\hat{p}_{1}=\frac{1-n_{1}\left(1-a_{1}\right) M_{1}}{1+n_{1} a_{i} M_{1}} \text {, de modo que }
$$

\footnotetext{
* Ultimo ano disponível até a elaboração deste trabalho.
} 
PAES, N.A. Mortalidade em Recife: aplicaçăo de um modelo de riscos competitivos. Rev. Saúde públ., S. Paulo, 19:251-62, 1985.

$\mathrm{n}_{\mathrm{i}}=$ intervalo de idade

$a_{i}=$ fator de separação* para a idade $i$

$\mathrm{M}_{\mathrm{i}}=$ coeficiente de mortalidade no intervalo de idade $\left(x_{1}, x_{1}+{ }_{n}\right)$

$D_{i}=$ número de óbitos ocorridos na idade $i$

$D_{i j}=$ número de óbitos ocorridos na idade i pela causa $\mathrm{j}$

A metodologia que usamos para a construção da Tábua de Vida de Múltiplo Decremento é análoga à da construção da tábua de vida, exceto na função que diz respeito a probabilidade de morte no intervalo de idade $\left(\mathrm{x}_{\mathrm{i}}, \mathrm{x}_{\mathrm{i}}+{ }_{1}\right)$, ${ }_{n} q_{x}$. Sendo assim, a probabilidade de morte que utilizamos é a líquida, ou seja, supomos que da população em estudo foi retirada uma causa (ou grupo de causas) específica de morte.

\section{RESULTADOS E DISCUSSÃA}

\section{Esperança de vida}

Para efeito de comparabilidade com a Tábua de Vida de Múitiplo Decremento, construímos tábuas de vida cujo resultado, para ambos os sexos, foi de 59,13 anos para a esperança de vida ao nascer. A vida média masculina foi de 55,43 anos e a feminina de 62,41 anos. A diferença entre os dois sexos, de 6,98 anos, revela um valor alto e que vern se acentuando gradativamente, nas últimas décadas, a favor das mulheres, de acordo com os dados de Drupell ${ }^{5}$ (1974). O total do ganho apresentado por este indicador, entre 1939 e 1969, foi de 26,71 anos para os homens e de 28,07 para as mulheres, ao passo que, entre 1969 e 1979, este mesmo ganho foi de 0,58 e de 1,87 anos, respectivamente, para homens e mulheres.

Apesar de que quanto mais alta é uma esperança de vida mais difíceis são os ganhos, admitimos que houve uma deteriorização nas condições de saúde do município de Recife, na última década.

De acordo com Acuña ${ }^{1}$ (1981), o município de Recife não alcançou a meta principal do Plano Regional de Saúde (Nações Unidas) para o período de 1971-80, o qual declarava que os governos da América Latina deveriam alcançar um ganho de cinco anos na esperança de vida para aqueles países que estavam abaixo dos 65 anos e recomendava, como instrumento principal para atingir este rível, a cobertura com serviços de saúde, água potável e saneamento básico a toda população atendida e subatendida.

A Assembléia Geral das Nações Unidas, ao proclamar o Terceiro Decênio para o Desenvolvimento e adotar a Estratégia Internacional do Desenvolvimento para o Decênio, estabeleceu, entre os principais objetivos dos países latinos, para o ano 2000 , alcançar, no mínimo, uma esperança de vida ao nascer de 70 anos. Se persistir esta tendência de ganhos na vida média, de acordo com as décadas passadas, dificilmente o município de Recife atingirá esta meta.

\section{Eliminação do grupo "Doenças \\ Infecciosas e Parasitárias"}

Este grupo foi responsável por $16,4 \%$ dos óbitos totais ocorridos no município de Recife, em 1979, sendo que 56,7\% dos óbitos desse grupo de causas ocorreram na faixa de 0-1 ano de idade. As doenças infecciosas e parasitárias representam, em números absolutos, o segundo grupo em importância para ambos os sexos, na mortalidade total.

As probabilidades de morte, sobrevivência e esperança de vida para este grupo, antes e depois de sua eliminação, encontram-se na Tabela 1.

\footnotetext{
* Os valores de separação usados foram encontrados por Oya ${ }^{14}$, para o distrito de São Paulo.
} 
PAES, N.A. Mortalidade em Recife: aplicaçũo de um modelo de riscos competitivos. Rev. Saúde públ., S. Paulo, 19:251-62, 1985.

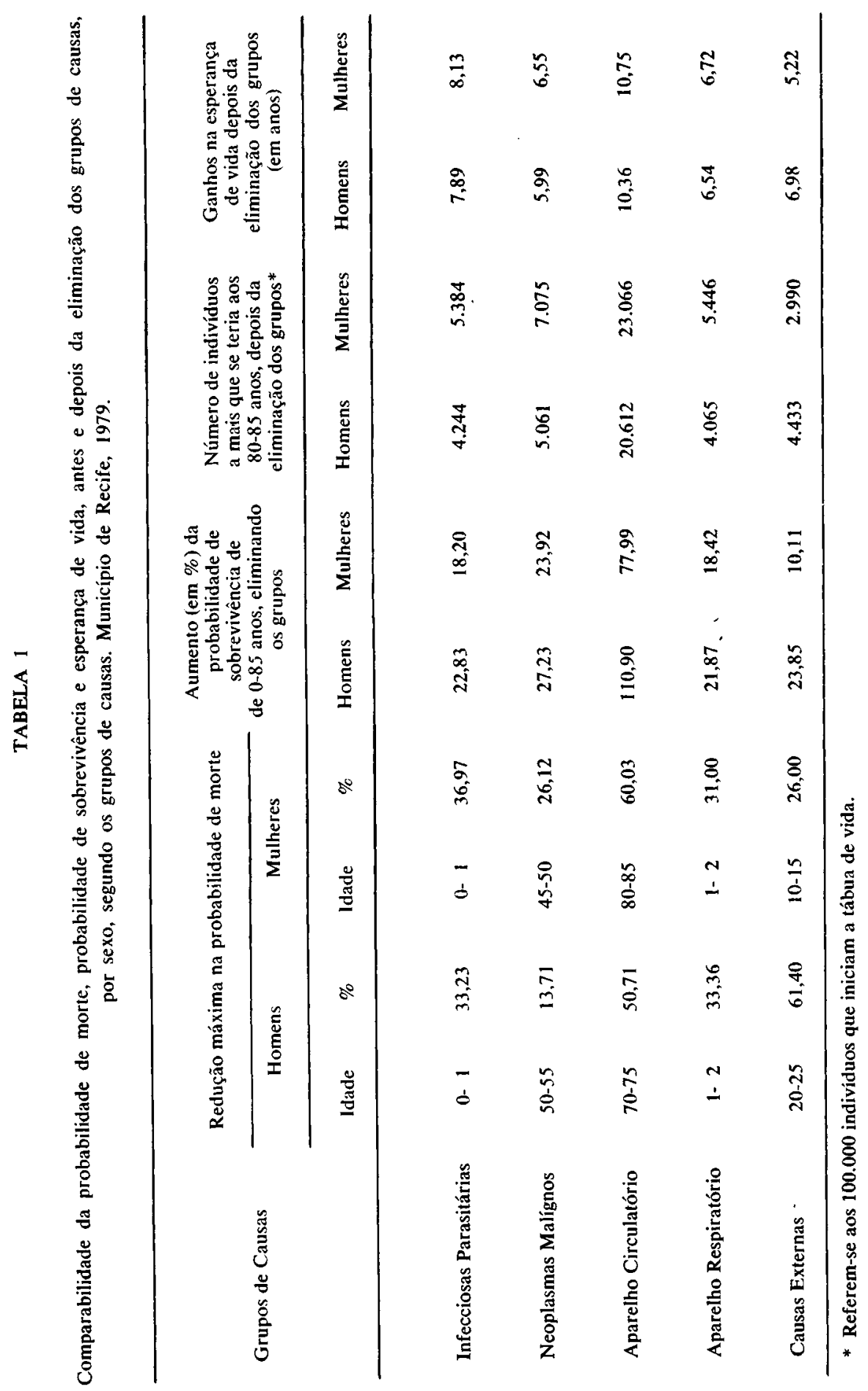


PAES, N.A. Mortalidade em Recife: aplicação de um modelo de riscos competitivos. Rev. Saúde públ., S. Paulo, 19:251-62, 1985.

A suposição de eliminação total das doenças infecciosas e parasitárias, como riscos de morte, é possível, se verificarmos a situação real de países desenvolvidos onde este grupo de causas tem uma representatividade nula ou quase nula, na mortalidade total, como ocorre, por exemplo, na Suécia, Islândia e Luxemburgo, de acordo com o World Health Statistics Annual ${ }^{20}$ (1980).

Estes níveis podem ser alcançados devido a estas causas serem, na sua totalidade, susceptíveis a medidas preventivas por saneamento básico, redutíveis por imunizações ou outros controles especiais.

As doenças infecciosas intestinais constituíram a principal causa básica de morte dentro do grupo das doenças infecciosas e parasitárias. Da mortalidade geral, ela foi responsável por $9,9 \%$ dos óbitos ocorridos.

Para avaliarmos o impacto das doenças infecciosas intestinais, na mortalidade geral, construímos Tábuas de Vida de Múltiplo Decremento com eliminação, apenas, deste subgrupo de causas. Caso elas não fossem um risco de morte, a esperança de vida para os homens seria acrescida de 6,42 anos e de 6,84 anos para as mulheres. Se levarmos em conta que este subgrupo de causas é plenamente evitável, apenas com este ganho, o município de Recife passaria para um nível de saúde bem mais próximo dos atingijos pelos países desenvolvidos.

A contribuição da deficiência nutricional na mortalidade infantil é outro importante fator ligado às doenças infecciosas e parasitárias. De acordo com a Organización Panamericana de la Salud ${ }^{15}$ (1971), dos óbitos de menores de 5 anos de idade, devido a doenças infecciosas como causa básica (excluídos os óbitos neonatais), $68,6 \%$ apresentavam a desnutrição como causa associada, em 1970.

A falta de serviços básicos, tais como água, esgotos e privadas, é importante fator que contribui para uma excessiva mortalidade, principalmente na infância, agravada ainda mais pela desnutrição e reduzido poder aquisitivo das classes sócio-econômicas menos privilegiadas da população.

Investigações realizadas neste sentido pela Organización Panamericana de la Salud registraram o município de Recife com baixíssimos níveis de serviços de saúde e o caracterizou como uma região que necessitava de "urgentes cuidados".

\section{Eliminação do grupo \\ "Neoplasmas Malígnos"}

O grupo de causas neoplasmas malígnos foi responsável por $8,2 \%$ dos óbitos totais e, em termos de proporção por faixa de idade, a maior incidência destes óbitos ocorreu dos 40 aos 75 anos de idade. Este grupo ocupou o quinto lugar na mortalidade dos homens e o quarto na mortalidade das mulheres.

Conforme podemos apreciar na Tabela 1 , o efeito do câncer se deu mais cedo nas mulheres chegando a atingir uma redução relativa de 7 vezes mais que nos homens, na faixa de 35-40 anos.

A população masculina suportou, portanto, um maior risco de morrer de câncer que as mulheres, mostrando estar ele associado, principalmente, às idades mais avançadas. Quando supomos a eliminação total do grupo de doenças neoplasmas malígnos, como risco de morte, sabemos que, com a tecnologia médica atual, esta suposição não é real. O que é possível, no entanto, é sua eliminação parcial.

Porém, o atual conhecimento a respeito deste grupo é suficiente para permitir a adoção de medidas capazes de prevenir algumas das formas mais frequientes do câncer. Para Stjernsward ${ }^{17}$ (1981), o conhecimento atual habilita-nos a predizer que, com medidas certas (a prevenção primária e secundária, recur- 
PAES, N.A. Mortalidade em Recife: aplicação de um modelo de riscos competitivos. Rev. Saúde públ., S. Paulo, 19:251-62, 1985.

sos suficientes e uma pesquisa bem orientada), pode-se evitar até um terço das formas de câncer existentes e curar até um terço dos casos.

\section{Eliminação do grupo \\ "Doenças do Aparelho Circulatório"}

Este grupo de causas foi responsável por $26,6 \%$ dos óbitos totais, a maior proporção de óbitos de todos os grupos de causas analisadas. Dentro do grupo, a participação masculina foi de $47,1 \%$ e a feminina foi de $52,9 \%$. As doenças do aparelho circulatório representaram, em número, o principal grupo de causas de morte no município, para ambos os sexos, com a maior predominância nas idades mais avançadas, conforme a $\mathrm{Ta}$ bela 1, que mostra a comparação deste grupo antes e depois da eliminação do mesmo, para as diversas medidas.

$\mathrm{Na}$ mortalidade total, o subgrupo das doenças cerebrovasculares representou $8,0 \%$ dos óbitos masculinos e $11,0 \%$ dos femininos. Devido a sua importância na mortalidade, construímos Tábuas de Vida de Múltiplo Decremento com exclusão deste subgrupo de causas como risco de morte. Estas tábuas mostraram que haveria um ganho de 6,23 anos na esperança de vida ao nascer para os homens e 6,57 anos para as mulheres.

De acordo com Carvalho e Ribeiro ${ }^{2}$ (1976), os países mais adiantados têm, nas doenças do aparelho circulatório, sua maior contribuição na mortalidade, associada, principalmente, ao envelhecimento. A tendência destes países é aumentar esta contribuição, uma vez que as causas elimináveis já deixaram de constituir problemas de saúde.

Diante das possibilidades médicas atuais, é irreal supor a eliminação total deste grupo de causas. Constitui-se em uma possibilidade a redução ou mesmo retardamento destas causas, fazendo com que elas ocorram em idades bem mais avançadas.

\section{Eliminação do grupo \\ "Doenças do Aparelho Respiratório"}

Este grupo foi responsável por 10,2\% dos óbitos totais, constituindo-se, em números absolutos, no terceiro grupo de causas mais importante na mortalidade dos residentes no município de Recife, em 1979. Destes óbitos, 37,0\% ocorreram antes de um ano de idade, o que revela o forte impacto deste grupo de causas, nesta idade.

Foi na infância e nas idades mais jovens que se deram as maiores reduções na probabilidade de morte, em termos relativos, para ambos os sexos; entretanto, conforme a Tabela 1 , o maior impacto se deu nos menores de dois anos, para ambos os sexos.

As doenças do aparelho respiratório tiveram uma representatividade importante no total de óbitos. Este grupo desperta mais atenção se considerarmos que algumas causas podem ser curáveis, como a pneumonia, para o qual foram registrados 201 óbitos. Participando com $7,1 \%$ dos óbitos totais foi a causa que mais matou neste grupo de causas. A pneumonia foi responsável por 59,0\% dos óbitos do grupo de causas do aparelho respiratório, sendo a causa que mais contribuiu para a mortalidade, depois das doenças infecciosas e parasitárias, de crianças menores de dois ${ }^{-}$anos de idade.

Para avaliarmos a importância da pneumonia como risco de morte, construímos Tábuas de Vida de Múltiplo Decremento e os resultados mostraram que, com a eliminação total desta causa, a esperança de vida ao nascer passaria para 61,42 anos para os homens e 68,48 anos para as mulheres, o que represen- 
PAES, N.A. Mortalidade em Recife: aplicação de um modelo de riscos competitivos. Rev. Saúde públ., S. Paulo, 19:251-62, 1985.

taria um ganho aproximado de 6 anos para ambos os sexos.

A elevação da esperança de vida dos indivíduos pode ser conseguida com 0 controle da tendência desse grupo de doenças, através do tratamento adequado dos casos já existentes, medidas preventivas, simplificação e padronização dos meios de diagnósticos, especial atenção às crianças abaixo de dois anos de idade e educação da população no sentido de maior participação individual e coletiva.

\section{Eliminação do grupo Causas Externas"}

Este grupo foi responsável por 8,3\% dos óbitos ocorridos no município de Recife, em 1979. Mais de $80,0 \%$ destes óbitos deram-se entre 5 e 60 anos, onde se concentra a população considerada ativa. A Tabela 1 revela o forte impacto da mortalidade masculina para esse grupo de causas, atingindo a redução máxima na probabilidade de morte, quase três vezes a mais que a redução máxima na probabilidade de morte das mulheres.

A mortalidade pelo grupo de causas externas se tem convertido em um problema não somente social, mas também de saúde pública. Este problema é muito significativo se tomarmos em consideração que este grupo afeta principalmente os adolescentes e os jovens adultos nos anos mais produtivos de sua vida. Este grupo respondeu por $21,0 \%$ dos óbitos totais, dos 10 aos 65 anos, constituindose, nesta faixa etária, a principal causa de mortalidade, em ambos os sexos.

Neste grupo, os acidentes de trânsito ocuparam especial importância, sendo responsáveis por $28,0 \%$ dos óbitos. Para Róman y Carrillo ${ }^{16}$ (1973) estes acidentes têm sido um crescente problema para a saúde pública e, observando que, à medida que o país se desenvolve, as taxas de acidentes aumentam, concluem alertando que os acidentes de trânsito aumentam em proporções geométricas com o número de veículos.

Parece arriscado se falar de eliminação ou erradicação completa desse grupo de causas, uma vez que para Róman e Carrillo são acontecimentos fortuitos ou provocados e, como tais, podem ocorrer quando e onde menos se espera, apesar de quantas providências se tomem.

E possível, no entanto, tomar medidas eficazes e eficientes, no sentido de minimizar as ocorrências das causas externas, através de programas educativos, primeiros socorros adequados, políticas e programas preventivos. Assim, os efeitos positivos seriam diretos e ganhos se obteriam principalmente na população considerada ativa.

\section{Considerações gerais e conclusão}

Depois de uma análise isolada daremos uma visão conjunta dos cinco grupos de causas, diferenciados por sexo e idade.

As Tabelas 2 e 3 mostram as diferenças relativas (\%) entre as probabilidades real e hipotética de morte. As maiores diferenças foram assinaladas para cada faixa etária, de modo que se formaram blocos de causas de morte.

E interessante observar que o grupo do aparelho respiratório somente representou a principal causa, para o sexo masculino, na faixa dos três anos de idade e o grupo dos neoplasmas malígnos, no sexo feminino, nas faixas etárias de 35-40 e 45-50 anos. Vale ressaltar a importância das causas externas para os homens e que o grupo das doenças do aparelho circulatório, em ambos os sexos, foi mais representativo em um maior número de faixas etárias. 
PAES, N.A. Mortalidade em Recife: aplicação de um modelo de riscos competitivos, Rev. Saúàe públ., S. Paulo, 19:251-62, 1985.

TABELA 2

Diferenças relativas (\%) entre as probabilidades real e líquida de morte, por grupos de causas de morte, segundo faixas etárias - sexo masculino. Município de Recife, 1979.

\begin{tabular}{cccccc}
\hline & \multicolumn{5}{c}{ Grupos de causas } \\
\cline { 2 - 6 } Idade & $\begin{array}{c}\text { Infecciosas e } \\
\text { parasitárias }\end{array}$ & $\begin{array}{c}\text { Neoplasmas } \\
\text { malígnos }\end{array}$ & $\begin{array}{c}\text { Aparelho } \\
\text { circulatório }\end{array}$ & $\begin{array}{c}\text { Aparelho } \\
\text { respiratório }\end{array}$ & $\begin{array}{c}\text { Causas } \\
\text { externas }\end{array}$ \\
\hline 0 & 33,23 & 0,07 & 0,21 & 13,99 & 0,92 \\
1 & 38,58 & 1,04 & 1,04 & 33,36 & 2,60 \\
2 & 34,58 & 0,00 & 0,00 & 32,66 & 7,68 \\
3 & 12,49 & 9,37 & 0,00 & 24,98 & 21,86 \\
4 & 25,91 & 7,40 & 3,70 & 22,21 & 11,10 \\
$5-10$ & 17,16 & 4,68 & 3,12 & 10,92 & 43,70 \\
$10-15$ & 5,35 & 3,56 & 5,35 & 3,56 & 60,67 \\
$15-20$ & 4,45 & 8,00 & 7,11 & 3,56 & 56,15 \\
$20-25$ & 9,95 & 3,06 & 4,59 & 4,59 & 61,40 \\
$25-30$ & 6,93 & 2,77 & 11,10 & 4,16 & 57,82 \\
$30-35$ & 13,95 & 2,93 & 8,07 & 5,13 & 41,23 \\
$35-40$ & 11,37 & 3,79 & 16,80 & 5,41 & 32,61 \\
$40-45$ & 11,01 & 9,10 & 21,12 & 3,83 & 21,12 \\
$45-50$ & 12,82 & 11,16 & 21,15 & 4,13 & 14,49 \\
$50-55$ & 9,01 & 13,71 & 32,00 & 6,00 & 8,01 \\
$55-60$ & 4,55 & 12,40 & 42,05 & 4,23 & 9,78 \\
$60-65$ & 6,51 & 10,61 & 44,21 & 5,96 & 4,87 \\
$65-70$ & 3,91 & 11,54 & 48,68 & 5,04 & 6,16 \\
$70-75$ & 3,18 & 11,81 & 50,71 & 3,98 & 3,44 \\
$75-80$ & 3,93 & 6,87 & 43,11 & 7,41 & 3,13 \\
$80-85$ & 2,82 & 8,63 & 43,67 & 8,20 & 2,42 \\
85 e + & - & - & - & - & - \\
\hline
\end{tabular}

TABELA 3

Diferenças relativas $(\%)$ entre as probabilidades real e líquida de morte, por grupos de causas de morte, segundo faixas etárias - sexo feminino. Município de Recife, 1979.

\begin{tabular}{|c|c|c|c|c|c|}
\hline \multirow[b]{2}{*}{ Idade } & \multicolumn{5}{|c|}{ Grupos de causas } \\
\hline & $\begin{array}{c}\text { Infecciosas e } \\
\text { parasitárias }\end{array}$ & $\begin{array}{c}\text { Neoplasmas } \\
\text { malígnos }\end{array}$ & $\begin{array}{c}\text { Aparelho } \\
\text { circulatório }\end{array}$ & $\begin{array}{c}\text { Aparelho } \\
\text { respiratório }\end{array}$ & $\begin{array}{l}\text { Causas } \\
\text { externas }\end{array}$ \\
\hline 0 & 36.97 & 0,00 & 0,08 & 14,44 & 0,65 \\
\hline 1 & 49,54 & 0,00 & 1,19 & 31,00 & 1,19 \\
\hline 2 & 34,85 & 0,00 & 2,32 & 30,20 & 9,29 \\
\hline 3 & 35,46 & 9,67 & 3,22 & 25,79 & 9,67 \\
\hline 4 & 37,49 & 0,00 & 0,00 & 12,50 & 0,00 \\
\hline $5 \cdot 10$ & 31,33 & 1,96 & 1,96 & 5,87 & 23,50 \\
\hline $10-15$ & 19,97 & 4,44 & 8,88 & 13,31 & 26,64 \\
\hline $15-20$ & 5,08 & 3,38 & 10,15 & 11,84 & 25,39 \\
\hline $20-25$ & 13,40 & 4,47 & 14,89 & 5,96 & 22,34 \\
\hline $25-30$ & 12,99 & 5,41 & 17,32 & 9,74 & 10,82 \\
\hline $30-35$ & 11,58 & 12,73 & 16,21 & 6,94 & 12,73 \\
\hline $35 \cdot 40$ & 10,54 & 21,10 & 18,46 & 9,66 & 10,54 \\
\hline $40-45$ & 10,19 & 22,60 & $2 \overline{8,45}$ & 5,09 & 8,74 \\
\hline $45-50$ & 9,70 & 26,12 & 22,46 & 5,45 & 9,09 \\
\hline $50 \cdot 55$ & 7,32 & 25,73 & $2 \overline{27,11}$ & 6,40 & 4,57 \\
\hline $55-60$ & 7,13 & 18,47 & 40,25 & 5,63 & 3,00 \\
\hline $60-65$ & 7,01 & 17,99 & 41,83 & 6,62 & 0,78 \\
\hline $65-70$ & 4,18 & 15,05 & 44,79 & 5,68 & 0,89 \\
\hline $70-75$ & 3,64 & 13,11 & 44,06 & 8,23 & 1,36 \\
\hline $75-80$ & 3,29 & 6,64 & 55,06 & 6,45 & 0,19 \\
\hline $80 e+$ & 2,89 & 4,83 & 60,03 & 7,11 & 1,28 \\
\hline
\end{tabular}


PAES, N.A. Mortalidade em Recife: aplicação de um modelo de riscos competitivos. Rev. Saúde públ., S. Paulo, 19:251-62, 1985.

A probabilidade de sobrevivência do nascimento até os 85 anos de idade, quando eliminamos os grupos de causas de morte (Tabela 4), mostra ter havido diferenças muito elevadas dessas causas, para ambos os sexos, principalmente para as mulheres.

\section{TABELA 4}

Probabilidade acumulada de sobreviver do nascimento aos 85 anos $(\%)$, por sexo, segundo grupos de causas de morte eliminados para o município de Recife, 1979.

\begin{tabular}{lcc}
\hline & $\begin{array}{c}\text { Probabilidade acumu- } \\
\text { lada de sobreviver } \\
(0-85) \%\end{array}$ \\
\cline { 2 - 3 } $\begin{array}{l}\text { Grupo de causas } \\
\text { eliminadas }\end{array}$ & Homens & Mulheres \\
\hline Sem eliminar causas & 11,2 & 20,8 \\
Infecciosas e parasitárias & 13,9 & 24,9 \\
Neoplasmas malígnos & 15,0 & 26,3 \\
Aparelho circulatório & 30,4 & 46,4 \\
Aparelho respiratório & 14,4 & 25,4 \\
Causas externas & 13,7 & 22,9 \\
\hline
\end{tabular}

A Tabela 1 mostra os ganhos (em anos) da esperança de vida ao nascer quando eliminamos os grupos de causas de morte discriminados por sexo. Segundo estes resultados, classificamos as principais causas de morte que atuaram sobre a população residente no município de Recife, em 1979. A ordenação mostra que:

i) no sexo masculino

1) doenças do aparelho circulatório

2) doenças infecciosas e parasitárias

3) causas externas

4) aparelho respiratório

5) neoplasmas malígnos ii) no sexo feminino

1) doenças do aparelho circulatório

2) doenças infecciosas e parasitárias

3) doenças do aparelho respiratório

4) neoplasmas malígnos

5) causas externas

De acordo com as considerações feitas podemos concluir que o nível de saúde da população residente no município de Recife, em 1979, mostrou-se contraditório ao apresentar taxas elevadas de óbitos por doenças degenerativas (doenças do aparelho circulatório e tumores), próprias de regiões desenvolvidas e, também, de óbitos por doenças infecciosas e parasitárias relacionadas com regiōes pouco desenvolvidas, apontando a existência de desigualdades dentro da mesma região, com uma população com características distintas, por um lado com bom nível de saúde e, por outro, em condições sanitárias desfavoráveis.

O caráter não homogêneo do nível de saúde da população residente do município tem suas origens no processo histórico dos fatores sócio-econômicos que privilegiam certas camadas da população em detrimento de outras. E neste contexto que as análises desse trabalho sugerem uma continuidade na investigação, no sentido de tentar explicar ou levantar hipóteses sobre os motivos que levaram a ocorrência dos grupos de causas estudadas, em certos extratos da população, em vista da sua importância no planejamento do setor saúde.

\section{AGRADECIMENTO}

A Maria Eliete Santiago, pelo permanente incentivo e apoio técnico na metodologia do trabalho. 
PAES, N.A. Mortalidade em Recife: aplicação de um modelo de riscos competitivos, Rev. Saúde públ., S. Paulo, 19:251-62, 1985.

PAES, N.A. [Mortality in Recife: application of a competitive risks model]. Rev. Saúde públ., S. Paulo, $19: 251-62,1985$.

ABSTRACT: The methodology of Chiang for the construction of the Decrement of Multiple Life Tables as applied to the resident population of the municipality of Recife, PE, Brazil, in 1979, is presented so as to evaluate the magnitude of the incidence of some groups of causes of death, according to the probability of death, survival and life expectancy. The average life for men was 55.43 years and for women 62.41 years. The total elimination of the groups of causes, as agents of the risk of death created the following gains in life expectancy, for men and women respectively: infection and parasitic diseases (7.9 and 8.1 years), malignant neoplasms (6.0 and 6.6 years), cardiovascular diseases (10.4 and 10.8 years), respiratory diseases (6.5 and 6.7 years) and external causes (7.0 and 5.2 years). It is concluded that the standard of health of the municipality of Recife showed, in 1979, a social and economical picture that was not homogenous, with a high mortality rate due to degenerative diseases characteristic of developing regions, and also of death caused by infection and parasitic diseases common to little-developed regions.

UNITERMS: Mortality, Recife, PE, Brazil. Life table, multiple decrements. Competing risks. Life expectancy.

\section{REFERENCIAS BIBLIOGRÁFICAS}

1. ACUÑA, N.H. Salud y desarrollo. Bol. Ofic. sanit. panamer., 91:95-8, 1981.

2. CARVALHO, A.V.W. de \& RIBEIRO, E.M. Estudo da mortalidade proporcional, segundo grupos de idade e causas de óbito em algumas capitais brasileiras, em 1970. Rev. bras. Estat., 37:457-82, 1976.

3. CHIANG, C.L. Introduction to stochastic process in biostatistics. New York, John Wiley, 1968. (Wiley Series in Probability and Mathematical Statistics).

4. CHIANG, C.L. Competing risks and conditional probability. Biometrics, 25:767. 76,1970 .

5. DRUPPEL, E. O nível da mortalidade no Recife em 1970. Rev. pernamb. Desenv., Recife, 1(2):153-65, 1974.

6. FUNDAÇÃO IBGE. Censo demográfico: Pernambuco. Rio de Janeiro, 1970. v. 1, t.10. (8.0 Recenseamento geral - Série Regional, 1970).

7. FUNDAÇÃO IBGE. Tabulações avançaçadas do censo demográfico. Rio de $\mathrm{Ja}$ neiro, 1981. v. 1, t. 2. $\left(9 .^{\circ}\right.$ Recenseamento geral do Brasil. Resultados preliminares, 1980).
8. GOTLIEB, S.L.D. Mortalidade diferencial por causas, São Paulo, Brasil, 1970: tábuas de vida de múltiplo decremento. Rev. Saúde públ., S. Paulo, 15:401-17, 1981.

9. GURALNICK, L. Some problems in the use of multiple causes of death. J. chron. Dis., 19:979-90, 1966.

10. HAKULINEN, T. Net probalities in theory of competing risks. Scand. actuarial $J$., p. $65-80,1977$.

11. KEYFITZ, N. What difference would it make if cancer were eradicated? An examination of the Taeuber Paradox. Demograph, 14: 411-8, 1977

12. MINISTERIO DA SAUDE. Divisão Nacional de Epidemiologia. Lista brasileira para mortalidade. Brasília, 1980.

13. MOESCHERGER, M.L. \& DAVID, H.A. Life tests under competing causes of failure and the theory of competing risks. Biometrics, 27:909-33, 1971.

14. OYA, D.R.T. Estudo da distribuição do fator de separação $f_{x}^{\prime}$ na tábua de sobrevivência. São Paulo, 1970. [Dissertação de Mestrado - Faculdade de Saúde Pública da USP] 
PAES. N.A. Mortalidade em Recife: aplicação de um modelo de riscos competitivos, Rev, Saúde pibl., S. Paulo, 19:251-62, 1985.

15. ORGANIZACION PANAMERICANA DE LA SALUD. Investigación intera. mericana de mortalidad en la niñez. Washington, D.C., 1971.

16. ROMAN y CARRILLO, G. L.A epidemiologia de los accidentes del transito. Bol. Ofic. sanit. panamer., 75:53-614. 1973.

17. STJERNSWARD, J. Pode-se evitar o câncer? Suúde do Mundo, p. 2.7. set./out. 1981.

18. TSAJ, S.P.; LEE, E.S.; HARIJY, R.J. The effect of a reducion in leading causes of death: potencial gains in life expectancy. Amer. J. publ. Hlth, 68:966-71, 1978.

19. WONG, O. A competing-risk model based on the life table procedure in epidemiological studies. Int. J. Epidem., 6: $153-9,1977$.

20. WORLD HEALTH STATISTICS ANNUAL: Vital Statistics and Causes of Dearl. (World Health Organization) Geneva, 1980.

Recebido para publicação em 10/10/1984

Reapresentado em 26/02/1985

A provado pura publicação em 26/04/J985 